\title{
El desempeño laboral en la municipalidad provincial de Rioja, región San Martin-2020
}

\author{
Gloria Marrufo Fernández \\ marrufofernandezg@gmail.com \\ Universidad Cesar Vallejo \\ ORCID: 0000-0002-8784-1894 \\ Jimmy Pacherrez Riva \\ rivapm@hotmail.com \\ Universidad Nacional de San Martin \\ ORCID: 0000-0002-8235-6371
}

\section{RESUMEN}

La presente investigación tuvo como objetivo identificar el nivel de rendimiento laboral de los trabajadores nombrados de la Municipalidad provincial de Rioja, región San Martín, 2020. Es un estudio fue de tipo no experimental, con diseño descriptivo propositiva. La muestra lo conformaron 105 trabajadores contratados, como instrumento de recolección de datos se utilizó el cuestionario. Los resultados de la variable rendimiento laboral, determinó desde la percepción del funcionario y colaborador, el nivel es "regular" en un 77.8\% (7) y 78.1\%(82), seguido de "deficiente con "11.1\%(1) y 17.1(18) y “eficiente en un "11.1\%(1) y 4.8\% (5). Concluyendo que existe un regular rendimiento laboral en los colaboradores nombrados, lo que demuestra que no están cumpliendo eficazmente con sus funciones; los directivos de la Municipalidad provincial de rioja debería buscar estrategias para motivar al personal y de esa manera mejorar el rendimiento laboral.

Palabras clave: Rendimiento laboral; eficiencia; eficacia; valores. 


\title{
Labor performance in the provincial municipality of Rioja, San
}

Martin region-2020

\begin{abstract}
The present research aimed to identify the level of labor performance of the named workers of the Provincial Municipality of Rioja, San Martín region, 2020. It was a nonexperimental study, with a descriptive propositional design. The sample was made up of 105 contracted workers, as a data collection instrument the questionnaire was used. The results of the variable work performance, determined from the perception of the official and collaborator, the level is "regular" in $77.8 \%$ (7) and $78.1 \%$ (82), followed by "deficient with" $11.1 \%$ (1) and 17.1 (18) and "efficient" $11.1 \%$ (1) and 4.8\% (5). Concluding that there is a regular job performance in the appointed collaborators, which shows that they are not effectively fulfilling their functions; The directors of the Provincial Municipality of Rioja should seek strategies to motivate staff and thus improve job performance.
\end{abstract}

Keywords: Labor performance; efficiency; effectiveness; values.

Artículo recibido: 03 nov. 2020 Aceptado para publicación: 07 dic. 2020 Correspondencia marrufofernandezg@gmail.com Conflictos de Interés: Ninguna que declarar 


\section{INTRODUCCIÓN}

Los nuevos desafíos mundiales de la economía en general y de la administración de recursos humanos en específico, pueden ilustrarse mediante los efectos que derivan de los convenios internacionales que muchos países han realizado en el curso del último decenio, por ejemplo, el tratado del libre comercio entre Canadá, Estados Unidos y México, que integran la economía de los 3 países, la obligación de las corporaciones como el administrador de recursos humanos para revisar casi la totalidad de su estrategia operativa.

En las empresas públicas de prestación de servicios, entre las cuales se encuentran las empresas municipales, un aspecto importante en ellas es la administración de los recursos humanos, entendida como la tarea que consiste en medir la relación de causa y efecto de diversos programas y políticas de recursos humanos en el resultado del último análisis financiero de la empresa.

En la Municipalidad provincial de Rioja, región San Martín existen deficiencias con la gestión de recursos humanos, no existe un buen liderazgo que sea el aparato propulsor para que tenga éxito la gestión, los funcionarios no saben llegar a sus subordinados para que estos se comprometan con la institución, otro problema que se presenta en la municipalidad es la falta de coordinación entre las operaciones y la estrategia por parte de los jefes de área, el personal que labora en la municipalidad no presenta predisposición para asumir los nuevos, presentando un nivel de rendimiento laboral regular.

Álvarez, B., Alfonso, D. \& Indacochea, B. (2018), El desempeño laboral: Un problema social de la ciencia. (artículo científico). Universidad del Pinar del Río, Cuba. Tipo de estudio cualitativo, diseño no experimental, población y muestra documentación, técnica análisis de casos, instrumento ficha de registro. Llegó a la conclusión, que, las formas y estilos de selección de los tomadores de decisiones en las entidades públicas no tributan a un liderazgo en sus organizaciones, de manera que estas cumplan efectivamente con el encargo social. El Estado debe ajustar la normativa jurídica a la realidad social, en el cual las Instituciones Públicas como privadas, reflejen una remuneración justa y equitativa, con el objetivo de alcanzar un salario digno, consagrado también en la Constitución. La verdadera importancia de la evaluación del Talento Humano requiere de una combinación correcta de conocimientos y habilidades para el desempeño laboral del personal y así ayudar a motivar el mejoramiento continuo de la institución con la sociedad. 
Para, Robbins y Judge (2013), expuso que: La evaluación del rendimiento en el trabajo es un sistema que provee a la organización retroalimentación y que influye en el incremento de las capacidades y habilidades del trabajador, también tiene como fin ayudar en las decisiones de quienes merecen un ascenso, aumento de sueldo o despido. (p. 555). Se entiende que, el rendimiento en el trabajo beneficia a los funcionarios y a las organizaciones, en el cual se aplican para la toma de decisiones administrativas, en el desarrollo y en la retroalimentación. Para evaluar el desempeño laboral, se utiliza la técnica del cuestionario tanto en el personal como los miembros de la directiva.

Importancia de conocer el rendimiento laboral. Según, Gerolamo (2003) fundamentó, que es importante conocer el desempeño laboral y se puede destacar cuatro como las categorías genéricas, conocidas como: (a) Confirmar posición: necesidad de tener información de desempeño para la toma de acciones gerenciales; (b) Comunicar posición: las organizaciones utilizan medidas de rendimiento para comunicar resultados a las partes interesadas; (c) Confirmar las prioridades: a través de la medición del rendimiento, es posible evaluar cuán lejos se está de las metas de rendimiento preestablecido; (d) Compilar el progreso: la medición de rendimiento en sí no mejora el mismo, pero hace el progreso explícito.

El proceso de medición de rendimiento posibilita el monitoreo de las variables y el seguimiento de los procesos, lo que permite: la anticipación de acciones y la toma de medidas preventivas, la resolución de problemas por medio de acciones correctivas, el aumento de eficiencia y eficacia de los procesos, por medio de medidas de mejora continua; la implantación de medidas innovadoras. Conocer el desempeño sirve como subsidio para las tomas de decisiones y determinación de estrategias de corto, medio y a largo plazo. El análisis de los resultados operativos permite a las empresas evaluar alternativas para mejorar las capacidades internas, así como prever las inversiones necesarias en personal, sistemas y procedimientos, con el fin de mejorar el rendimiento futuro. Por lo tanto, conocer el desempeño laboral permite la búsqueda de la mejora continua de los servicios y de la productividad organizacional, aumentando la satisfacción de los clientes, la competitividad empresarial y la participación en el mercado.

Indicadores de rendimiento laboral. Son atributos esenciales en los procesos de la organización. Estos permiten que las evaluaciones se realicen sobre la base de datos e información cualitativa / cuantitativa, dando mayor fiabilidad de las conclusiones. Según 
Kardec (2002), ellos pueden ser representados por índices, coeficientes, tasas, parámetros, porcentaje o valores cualitativos. Los indicadores son importantes para la planificación y el control de los procesos de las organizaciones. (p. 9). Es así, que el escrutado de los resultados a través de indicadores es fundamental para el análisis y crítica del desempeño de la organización en relación con las metas establecidas, subsidiando las tomas de decisión en los diversos niveles de la empresa y la replantación de las actividades (Takashina, 1996, p. 11).

Estas metas a ser establecidas pueden ser internas (por medio de previsiones basadas en series históricas o según la expectativa de la gerencia) o externas, con el uso de benchmarking (utilizando valores de los competidores, los valores medios del mercado y / o mejores referencias de rendimiento). De lo anterior, se observa que la evaluación del rendimiento es la determinación de indicadores de desempeño y se utilizará de forma que estén bien alineados con los objetivos de la empresa. Es fundamental que los indicadores representen características traducidas de las necesidades y expectativas de todas las perspectivas seleccionadas, siempre teniendo en cuenta los objetivos y las estrategias de la organización y referenciales externos de comparación.

Para que los indicadores puedan ayudar eficazmente a la toma de decisiones de decisión, es imperativo que sean de fácil obtención, comprensión, comparación y no sean ambiguos (Lima, 2001). Un indicador debe crearse, de forma que garantice la disponibilidad de los datos y resultados más relevantes en el menor tiempo posible y al menor costo. (p.

Álvarez, B., Alfonso, D. \& Indacochea, B. (2018), El desempeño laboral: Un problema social de la ciencia. (artículo científico). Universidad del Pinar del Río, Cuba. Tipo de estudio cualitativo, diseño no experimental, población y muestra documentación, técnica análisis de casos, instrumento ficha de registro. Llegó a la conclusión, que, las formas y estilos de selección de los tomadores de decisiones en las entidades públicas no tributan a un liderazgo en sus organizaciones, de manera que estas cumplan efectivamente con el encargo social.

El Estado debe ajustar la normativa jurídica a la realidad social, en el cual las Instituciones Públicas como privadas, reflejen una remuneración justa y equitativa, con el objetivo de alcanzar un salario digno, consagrado también en la Constitución.

En cambio, Vera y Suárez (2018), Incidencia del clima organizacional en el desempeño laboral, el servicio al cliente; Corporación de Telecomunicaciones del Cantón la 
Libertad. (artículo científico). Universidad Estatal Península de Santa Elena. República del Ecuador. Tipo de investigación básica, diseño no experimental, población y muestra 100 funcionarios, técnica encuesta, instrumento cuestionario; donde concluyeron, que, es insuficiente el reconocimiento por parte de la empresa, influye en la autoestima del empleado, debe de dar iniciativa para que exista satisfacción laboral y aumentar la productividad de la empresa. En este sentido el $80 \%$ indicó que se sienten desmotivados los trabajadores porque no se les reconoce su esfuerzo a través de incentivos, vacaciones, bonos, etc.

Ales (2002) definió al rendimiento laboral como: el desenvolvimiento personal y profesional del empleado, demostrando a través de sus funciones la obtención de óptimos resultados en la institución, así como el buen manejo de los recursos instituciones, garantizando un ambiente formidable entre trabajadores. (p. 27); en cambio, Whitmore (2002) expresó que “El rendimiento laboral consiste en mirar más allá de lo que se espera y establecer normas más altas que superen la expectativa y generen resultados satisfactorios". (p. 105)

Para, Robbins y Judge (2013), expuso que: La evaluación del rendimiento en el trabajo es un sistema que provee a la organización retroalimentación y que influye en el incremento de las capacidades y habilidades del trabajador, también tiene como fin ayudar en las decisiones de quienes merecen un ascenso, aumento de sueldo o despido. (p. 555). Se entiende que, el rendimiento en el trabajo beneficia a los funcionarios y a las organizaciones, en el cual se aplican para la toma de decisiones administrativas, en el desarrollo y en la retroalimentación. Para evaluar el desempeño laboral, se utiliza la técnica del cuestionario tanto en el personal como los miembros de la directiva.

Según Melinkoff (1969) los "modelos de estructuras organizacionales en términos generales, un "modelo" es la representación de alguna cosa, o como lo definió Jorge Etkin" (p. 90). En el sentido más general se llama modelo a un conjunto de elementos, sean estos materiales, símbolos gráficos, ideas o palabras que son utilizadas para representar un fenómeno real con una finalidad práctica; se pueden clasificar los modelos de estructuras organizacionales en cuatro clases ó tipos fundamentales; la competencia existente, para la producción de más y mejores bienes y servicios, ha provocado que las organizaciones se preocupen cada vez más, por eficientes los procedimientos administrativos, los procesos productivos y en general las estructuras organizacionales. 
Dimensiones del desempeño laboral. Son tres: (i)

Demanda de tareas: se evalúa él trabajo individual del empleado, se basa en cuanto produce, el tiempo que le toma y los costos que genera.

Valores: son las características que tiene cada trabajador no necesariamente son las que estén enfocadas con el trabajo que desempeñan sino propias del empleado.

Productividad: son criterios enfocados únicamente con el trabajo que desempeñan como por ejemplo experiencia, bueno resultado.

\section{MATERIALES Y MÉTODOS}

El trabajo científico recurrió al método lógico deductivo, de manera tal, que se llevó a cabo la aplicación de los principios teóricos sobre el rendimiento laboral para analizar y determinar las caracterizaciones resaltantes de los nudos críticos de las dimensiones demanda de tareas, valores y productividad en la municipalidad provincial de Rioja. La población conformada por 114 entre trabajadores y funcionarios y muestra de 105 trabajadores y 9 funcionarios de las gerencias cargos de cconfianza entre ellos Gerencia Municipal, Gerencia de Planificación y Presupuesto, Gerencia de Administración, Gerencia de Desarrollo Económico Local, Gerencia de Desarrollo Ambiental, Gerencia de Seguridad Ciudadana, Gerencia de Maquinaria y Equipo y Oficina de Asesoría Jurídica. Se excluyeron a los colaboradores nombrados. Se aplicó un cuestionario para caracterizar la demanda de tareas, valores y productividad de los colaboradores nombrados en la entidad, así el nivel del rendimiento laboral. Para el grado de confiabilidad se utilizó el Alfa de Cronbach.

\section{RESULTADOS Y DISCUSIÓN}

El resultado arrojado de la investigación se obtuvo por la aplicación del cuestionario a los colaboradores y funcionarios de la municipalidad provincial de Rioja, así mismo, se realizó el análisis e interpretación de la información obtenida.

\section{Tabla 1}

Nivel de rendimiento laboral de los colaboradores

\begin{tabular}{lccccc}
\hline \multirow{2}{*}{ Nivel } & \multirow{2}{*}{ Escala } & \multicolumn{2}{c}{ Funcionario } & \multicolumn{2}{c}{ Colaborador } \\
\cline { 3 - 6 } & & fi & \% & fi & $\%$ \\
\hline Eficiente & $44-60$ & 1 & $11.1 \%$ & 5 & $4.8 \%$ \\
Regular & $28-43$ & 7 & $77.8 \%$ & 82 & $78.1 \%$ \\
Deficiente & $12-27$ & 1 & $11.1 \%$ & 18 & $17.1 \%$ \\
\hline
\end{tabular}




\begin{tabular}{lllll}
\hline Total & 9 & $100.0 \%$ & 105 & $100.0 \%$
\end{tabular}

Fuente: cuestionario aplicado a funcionarios y colaboradores contratados, municipalidad de Rioja

\section{Interpretación}

El nivel de rendimiento laboral de los colaboradores desde la percepción del funcionario es "regular" en un 77,8\% (7), seguido de "deficiente" y "eficiente" en un 11,1\% (1), respectivamente.

Del mismo modo, los mismos colaboradores opinan que su rendimiento es "regular" en un $78.1 \%$ (82), seguido de "deficiente" en un 17,1\% (18) y "eficiente" en un 4,8\% (5).

\section{Tabla 2}

Rendimiento laboral desde la percepción del funcionario

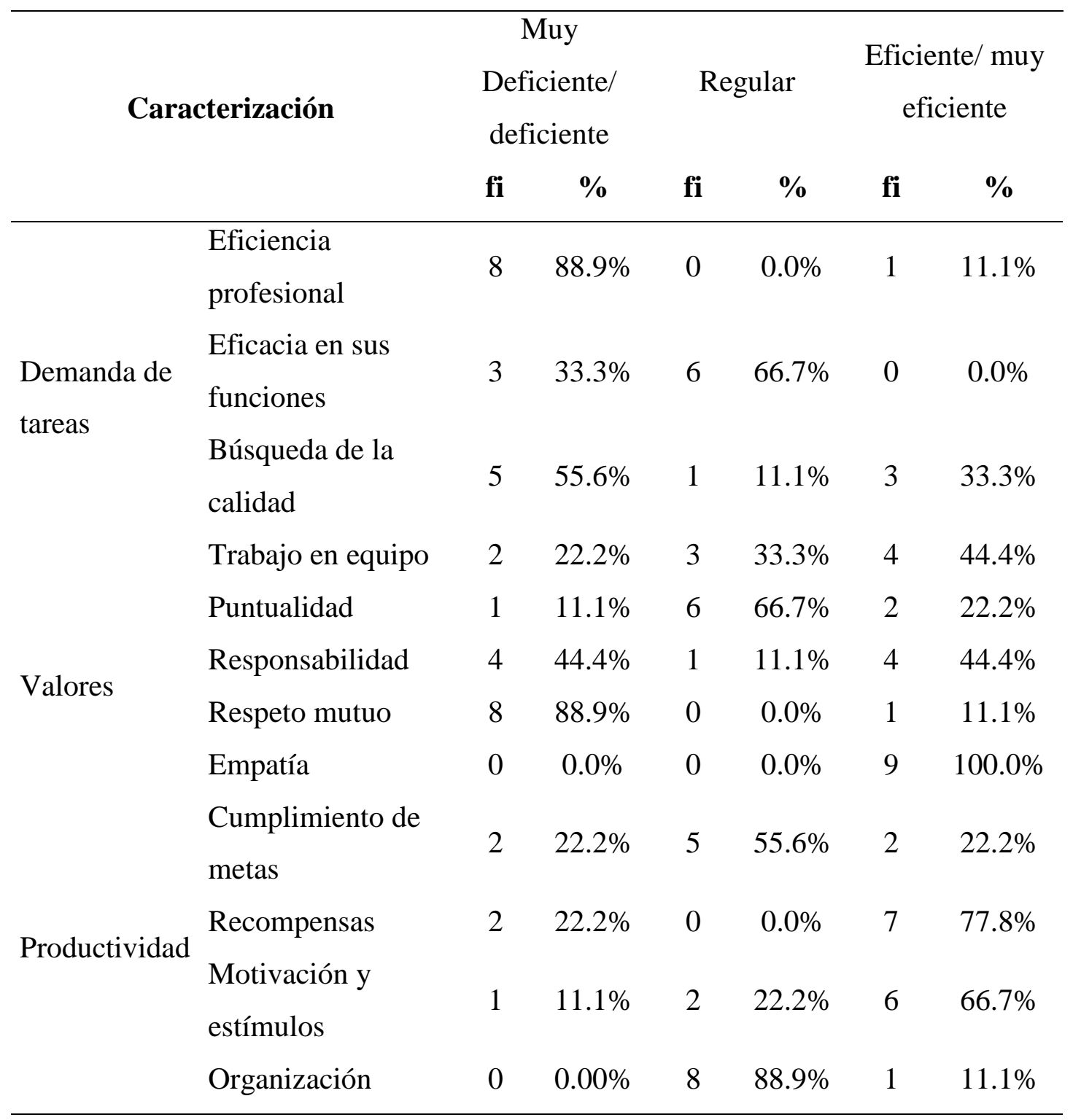

Fuente: cuestionario aplicado a funcionarios de la, municipalidad de Rioja 


\section{Interpretación.}

La tabla 2 muestra la caracterización del rendimiento laboral desde la percepción del funcionario, evidenciándose que en la dimensión demanda de tareas, los colaboradores nombrados cumplen "muy deficiente y deficientemente" con sus funciones en un $88,9 \%$ (8), no brindan un servicio de calidad a la población en un 55,6\% (5). Asimismo, consideran que el desarrollo de sus funciones e manera eficaz es "regular" en un 66,7\% (6).

En la dimensión valores, los funcionarios consideran que es deficiente y muy deficiente en un $88.9 \%$ (8) el respeto ante las opiniones de sus demás compañeros; sin embargo, opinan que se relacionan con mucha facilidad con los demás colaboradores siendo eficiente y muy eficiente en un $100 \%$ (9)

Finalmente, en la dimensión productividad los funcionarios opinan que los colaboradores nombrados cumplen de manera regular en un 55.6\% (5) el trabajo relacionado a las metas del programa de incentivos municipales, así mismo cumplen de manera Eficiente y muy eficiente en un $77.8 \%$ (7) el trabajo relacionado al total de metas institucionales planificadas durante el ejercicio fiscal, finalmente también opinan que los colaboradores nombrados buscan estrategias para el cumplimiento de sus tareas y aplicar una correcta gestión del tiempo de una manera regular en un $88.9 \%$ (8) .

\section{Tabla 3}

Rendimiento laboral desde la percepción del colaborador

\begin{tabular}{|c|c|c|c|c|c|c|c|}
\hline \multirow{2}{*}{\multicolumn{2}{|c|}{ Caracterización }} & \multicolumn{2}{|c|}{$\begin{array}{c}\text { Muy } \\
\text { Deficiente/ } \\
\text { deficiente }\end{array}$} & \multicolumn{2}{|c|}{ Regular } & \multicolumn{2}{|c|}{$\begin{array}{c}\text { Eficiente/ muy } \\
\text { eficiente }\end{array}$} \\
\hline & & fi & $\%$ & $\mathbf{f i}$ & $\%$ & fi & $\%$ \\
\hline \multirow{4}{*}{$\begin{array}{l}\text { Demanda de } \\
\text { tareas }\end{array}$} & $\begin{array}{l}\text { Eficiencia } \\
\text { profesional }\end{array}$ & 28 & $26.7 \%$ & 56 & $53.3 \%$ & 21 & $20.0 \%$ \\
\hline & $\begin{array}{l}\text { Eficacia en sus } \\
\text { funciones }\end{array}$ & 47 & $44.8 \%$ & 41 & $39.0 \%$ & 17 & $16.2 \%$ \\
\hline & $\begin{array}{l}\text { Búsqueda de la } \\
\text { calidad }\end{array}$ & 46 & $43.8 \%$ & 30 & $28.6 \%$ & 29 & $27.6 \%$ \\
\hline & Trabajo en equipo & 57 & $54.3 \%$ & 15 & $14.3 \%$ & 33 & $31.4 \%$ \\
\hline Valores & Puntualidad & 22 & $21.0 \%$ & 28 & $26.7 \%$ & 55 & $52.4 \%$ \\
\hline
\end{tabular}




\begin{tabular}{llllllll}
\hline & Responsabilidad & 40 & $38.1 \%$ & 27 & $25.7 \%$ & 38 & $36.2 \%$ \\
& Respeto mutuo & 53 & $50.5 \%$ & 25 & $23.8 \%$ & 27 & $25.7 \%$ \\
& Empatía & 42 & $40.0 \%$ & 39 & $37.1 \%$ & 24 & $22.9 \%$ \\
& Cumplimiento de & & & & & & \\
& metas & 56 & $53.3 \%$ & 30 & $28.6 \%$ & 19 & $18.1 \%$ \\
Productividad & Recompensas & 64 & $61.0 \%$ & 17 & $16.2 \%$ & 24 & $22.9 \%$ \\
& Motivación y & & & & & & \\
& estímulos & 88 & $83.8 \%$ & 7 & $6.7 \%$ & 10 & $9.5 \%$ \\
& Organización & 48 & $45.7 \%$ & 28 & $26.7 \%$ & 29 & $27.6 \%$ \\
\hline
\end{tabular}

Fuente: cuestionario aplicado a colaboradores contratados, municipalidad de Rioja

\section{Interpretación.}

La tabla 3 muestra la caracterización del rendimiento laboral desde la percepción del colaborador, en la cual se evidencia que en la dimensión demanda de tareas, los colaboradores nombrados cumplen de manera "regular" con sus funciones en un 53.3\% (55), ni utilizan una correcta gestión del tiempo en el cumplimiento de sus tareas siendo deficiente y muy deficiente en un 54,3\% (57).

En la dimensión valores, los colaboradores consideran que es deficiente y muy deficiente en un $53.5 \%$ (53) el respeto ante las opiniones de sus demás compañeros

Finalmente, en la dimensión productividad los colaboradores opinan que los trabajadores nombrados son ineficientes y muy ineficientes en un $53.3 \%$ (56) y 61 . \% (64) con referencia al cumplimiento de metas del Programa de Incentivos y metas institucionales que la municipalidad tiene programada en sus instrumentos de gestión y tampoco buscan estrategias para el cumplimiento de sus tareas ni aplicar una correcta gestión del tiempo en un $83.8 \%(88)$.

\section{Tabla 4}

Gestión del cambio desde la percepción del funcionario

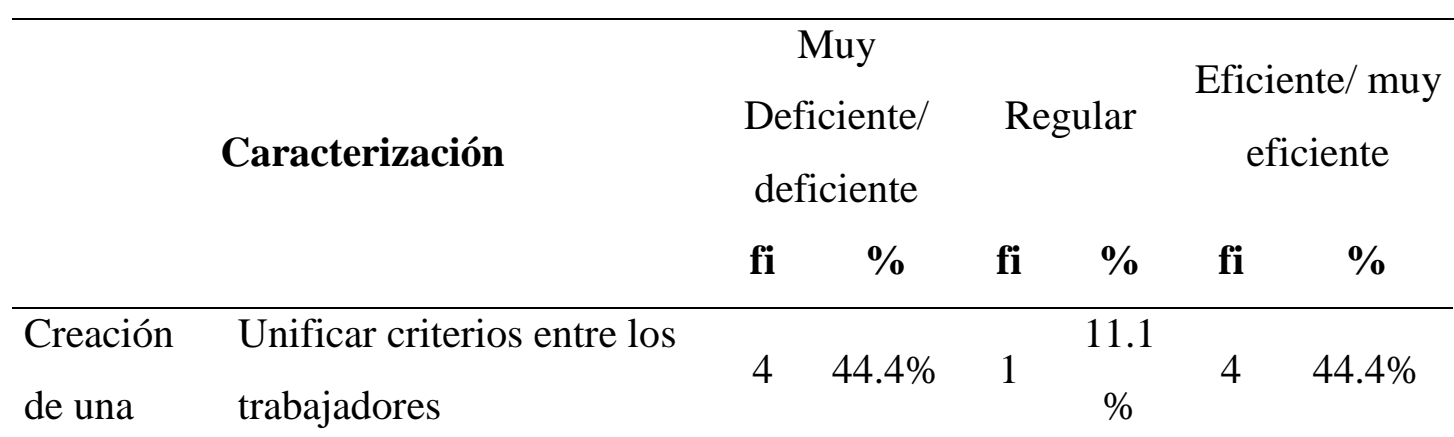




\begin{tabular}{|c|c|c|c|c|c|c|c|}
\hline \multirow[t]{2}{*}{$\begin{array}{l}\text { necesidad } \\
\text { en común }\end{array}$} & $\begin{array}{l}\text { Hacer entender que el } \\
\text { cambio es importante }\end{array}$ & 8 & $88.9 \%$ & 1 & $\begin{array}{l}11.1 \\
\%\end{array}$ & 0 & $0.0 \%$ \\
\hline & $\begin{array}{l}\text { Entender que la entidad es } \\
\text { importante. }\end{array}$ & 3 & $33.3 \%$ & 6 & $\begin{array}{c}66.7 \\
\%\end{array}$ & 0 & $0.0 \%$ \\
\hline \multirow{3}{*}{$\begin{array}{l}\text { Crear una } \\
\text { visión de } \\
\text { futuro }\end{array}$} & $\begin{array}{l}\text { Cambio en el } \\
\text { comportamiento }\end{array}$ & 2 & $22.2 \%$ & 6 & $\begin{array}{c}33.3 \\
\%\end{array}$ & 4 & $44.4 \%$ \\
\hline & Trabajadores motivados. & 3 & $33.3 \%$ & 3 & $\begin{array}{c}44.4 \\
\%\end{array}$ & 2 & $22.2 \%$ \\
\hline & $\begin{array}{l}\text { Trabajadores que ven el } \\
\text { impacto del cambio }\end{array}$ & 4 & $44.4 \%$ & 4 & $\begin{array}{c}55.6 \\
\%\end{array}$ & 0 & $0.0 \%$ \\
\hline \multirow{3}{*}{$\begin{array}{l}\text { Movilizaci } \\
\text { ón de los } \\
\text { implicados }\end{array}$} & $\begin{array}{l}\text { Promotores líderes del } \\
\text { cambio }\end{array}$ & 2 & $22.2 \%$ & 6 & $\begin{array}{c}66.7 \\
\%\end{array}$ & 1 & $11.1 \%$ \\
\hline & Ser autores del cambio & 3 & $33.3 \%$ & 4 & $\begin{array}{c}44.4 \\
\%\end{array}$ & 2 & $22.2 \%$ \\
\hline & Involucramiento. & 4 & $44.4 \%$ & 5 & $\begin{array}{c}55.6 \\
\%\end{array}$ & 0 & $0.0 \%$ \\
\hline \multirow{3}{*}{$\begin{array}{l}\text { Cambio de } \\
\text { sistemas y } \\
\text { estructuras }\end{array}$} & Gobierno Electrónico & 3 & $33.3 \%$ & 2 & $\begin{array}{c}22.2 \\
\%\end{array}$ & 4 & $44.4 \%$ \\
\hline & Asumir nuevos retos & 7 & $77.8 \%$ & 2 & $\begin{array}{c}22.2 \\
\%\end{array}$ & 0 & $0.00 \%$ \\
\hline & $\begin{array}{l}\text { Adaptarse a las } \\
\text { innovaciones. }\end{array}$ & 7 & $77.8 \%$ & 2 & $\begin{array}{c}22.2 \\
\%\end{array}$ & 0 & $0.00 \%$ \\
\hline \multirow{3}{*}{$\begin{array}{l}\text { Monitoreo } \\
\text { de avances }\end{array}$} & Seguimiento & 4 & $44.4 \%$ & 4 & $\begin{array}{c}44.4 \\
\%\end{array}$ & 1 & $11.1 \%$ \\
\hline & Evaluaciones & 9 & $\begin{array}{c}100.0 \\
\%\end{array}$ & 0 & $0.0 \%$ & 0 & $0.00 \%$ \\
\hline & Asesoramiento & 1 & $11.1 \%$ & 4 & $\begin{array}{c}44.4 \\
\%\end{array}$ & 4 & $44.4 \%$ \\
\hline
\end{tabular}

Fuente: cuestionario aplicado a funcionarios de la, municipalidad de Rioja

\section{Interpretación.}


La tabla 4 muestra la caracterización de la gestión del cambio desde la percepción del funcionario, en la cual se evidencia que en la dimensión demanda de tareas, del 100\% (9) de funcionarios que gerencia la municipalidad de Rioja, el 88,9\% (8) opina como "Muy deficiente y deficiente" que los colaboradores entiendan lo importante que es el cambio no colaboran en los mecanismos de mejora que plantea la entidad, así como la municipalidad muchos de ellos a un desconocen los objetivos institucionales la misión y la visión, pero de forma "Regular" en un $66,7 \%$ (6).

En la dimensión Crear una visión de futuro del 100\% (9) funcionarios opinan de manera regular en $55.6 \%$ (5) que los colaboradores nombrados estén preparados para los cambios institucionales; así mismo en la dimensión movilización de los implicados el 66.7\% (6) y $55.6 \%$ (5) opinan de manera regular sobre los colaboradores nombrados que demuestran capacidad de liderazgo para ejercer funciones en sus puestos, así como en involucramiento en la planificación y ejecución de metas institucionales.

En la Dimensión Cambio de sistemas y estructuras el 77.8\% (7) de funcionarios opinan deficiente y muy deficiente si los colaboradores nombrados serían capaces en asumir nuevos retos institucionales, así como a las adaptaciones y cambios tecnológicos dentro de la entidad

En la Dimensión Monitoreo de avances el 100\% (9) de los funcionarios que ocupan cargos gerenciales en la Municipalidad opinan que, los colaboradores nombrados no evalúan a su personal que tienen a su cargo para el desempeño de sus funciones

\section{Tabla 5}

Caracterización de la Gestión del cambio desde la percepción del Colaborador

\begin{tabular}{|c|c|c|c|c|c|c|c|}
\hline \multicolumn{2}{|c|}{ Caracterización } & \multicolumn{2}{|c|}{$\begin{array}{c}\text { Muy Deficiente/ } \\
\text { deficiente }\end{array}$} & \multicolumn{2}{|c|}{ Regular } & \multicolumn{2}{|c|}{$\begin{array}{c}\text { Eficiente/ muy } \\
\text { eficiente }\end{array}$} \\
\hline & & $\mathbf{f i}$ & $\%$ & fi & $\%$ & $\mathbf{f i}$ & $\%$ \\
\hline \multirow{4}{*}{$\begin{array}{l}\text { Creación de } \\
\text { una } \\
\text { necesidad en } \\
\text { común }\end{array}$} & $\begin{array}{l}\text { Unificar criterios } \\
\text { entre los } \\
\text { trabajadores }\end{array}$ & 33 & $31.4 \%$ & 55 & $52.4 \%$ & 17 & $16.2 \%$ \\
\hline & $\begin{array}{l}\text { Hacer entender } \\
\text { que el cambio es } \\
\text { importante }\end{array}$ & 50 & $47.6 \%$ & 39 & $37.1 \%$ & 16 & $15.5 \%$ \\
\hline & $\begin{array}{l}\text { Entender que la } \\
\text { empresa es } \\
\text { importante. }\end{array}$ & 45 & $42.9 \%$ & 32 & $30.5 \%$ & 28 & $26.7 \%$ \\
\hline & $\begin{array}{l}\text { Cambio en el } \\
\text { comportamiento }\end{array}$ & 34 & $32.4 \%$ & 25 & $23.8 \%$ & 46 & $43.8 \%$ \\
\hline
\end{tabular}




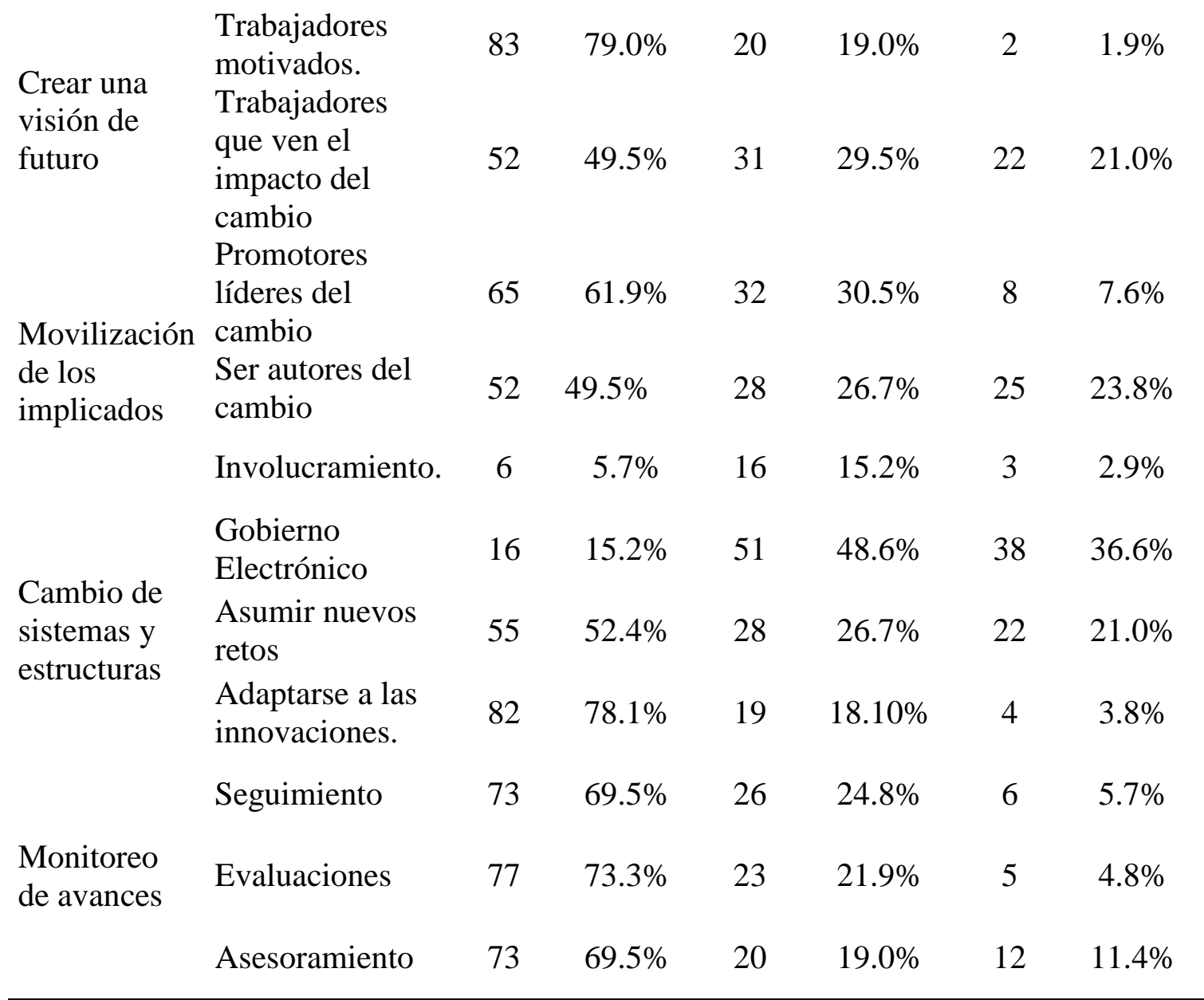

Fuente: cuestionario aplicado a colaboradores contratados, de la municipalidad de Rioja

\section{Interpretación.}

La tabla 5 muestra los resultados de la caracterización de la gestión del cambio desde la percepción del colaborador, en la cual se evidencia que en la dimensión crear una necesidad en común, del 100\% (105) de colaboradores de la municipalidad de Rioja, el $52.4 \%$ (55) opina de manera regular que los colaboradores nombrados utilicen el manual de normas políticas y procedimientos para el ejercicio de sus funciones.

En la dimensión Crear una visión de futuro el 79.0\% (86) colaboradores opinan de manera deficiente y muy deficiente que los colaboradores nombrados estén motivados por algún incentivo laboral por cumplimiento de metas, así mismo en la dimensión Movilización de los implicados el 61.9\% (65) colaboradores opinan de manera regular que el personal nombrado demuestren capacidad de liderazgo.

En la dimensión Cambio de sistemas y estructuras, el 52.4\% (55) y el 78.1\% (82) del $100 \%$ de los colaboradores opinan de manera regular que los colaboradores nombrados 
estén preparados para asumir nuevos retos y/o estén preparados para adaptarse a los cambios de innovación que la entidad plantee.

En la dimensión Monitoreo de avances del 100\% de colaboradores opinan de manera regular en un $69.5 \%$ (73), $73.3 \%$ (77) y $69.5 \%$ (73) referente al seguimiento de avances de metas institucionales por parte de la Gerencia de Planificación y Presupuesto así como también a la evaluación y asesoramiento que brindan los colaboradores nombrados al personal a su cargo.

\section{CONCLUSIÓN}

Los resultados del presente trabajo responden a los objetivos planteados siendo uno de los objetivos, identificar el nivel de rendimiento laboral de los colaboradores en la Municipalidad Provincial de Rioja, 2020. Según, la percepción del funcionario y del colaborador es "regular" (77,8\% Vs $78,1 \%)$ respectivamente; lo que evidencia la necesidad de mejorar su trabajo. La verdadera importancia de la evaluación del Talento Humano requiere de una combinación correcta de conocimientos y habilidades para el desempeño laboral del personal y así ayudar a motivar el mejoramiento continuo de la institución con la sociedad.

Según, Chávez (2018), el 75\% muestran puntajes promedios indicando que, el desempeño laboral depende mucho de las decisiones estratégicas que se tomen en la organización, ya que estas ayudan a identificar puntos críticos en el trabajo, determinan las tareas, realizan cambios en la estructura de la organización, influyen en las relaciones de los grupos y mejora notoriamente la eficacia de la organización; es por ello que el autor resalta que el éxito de la organización se basa fundamentalmente en la aplicación de estrategias correctas con los grupos de trabajo.

Del mismo modo, Vilcas (2019) muestra resultados similares, el 72\% manifestó que el desempeño laboral es regular, hace falta capacitación permanente, analizar el resultado de la evaluación del desempeño, procesar los resultados de productividad, tener en cuenta las observaciones de los supervisores, y realizar periódicamente evaluaciones de conocimientos y habilidades del puesto. Este autor, afirma que las posibles razones por las cuales el trabajador no logra cumplir con los estándares de desempeño son: dificultad de habilidades, conocimiento y/o inteligencia, capacidades de entendimiento $\mathrm{y}$ competencias, dificultades de esfuerzo, motivación para ejecutar las tareas y dificultades externas del trabajo, así como, bajas remuneraciones, deficientes condiciones de trabajo. 
En cuanto a la caracterización del rendimiento laboral de los colaboradores, en la dimensión demanda de tareas, la mayoría expresó que es deficiente, porque carecen de eficiencia profesional. Igualmente, en la dimensión valores, no son puntuales, poco responsables y no practican el respeto mutuo. En la dimensión productividad, buscan estimular e incentivar a los trabajadores.

Los resultados obtenidos del trabajo de investigación de, Seminario (2019), coinciden con los de este trabajo, en las dimensiones establecidas la mayoría mencionó que es deficiente, puesto que predomina más las debilidades que las fortalezas lo que viene a influir en el "bajo rendimiento laboral", estos factores se resumen en: escasa capacidad, poca motivación y inadecuado ambiente laboral, dichos factores pueden afectar de forma positiva o negativa el desempeño del individuo. Por ejemplo, la baja motivación en el personal, los problemas externos como cuestiones familiares y sociales afectan directamente en el cumplimiento de los objetivos propuestos provocando un desequilibrio y lentitud en el logro de las metas de la organización.

En cambio, Ayala y Pajuelo (2018), difiere con los resultados del estudio, reporta que el $63 \%$ menciona que el desempeño laboral es alto, a pesar que los trabajadores se encuentran poco motivados y poco valorados su desempeño en el trabajo es bueno; por ello, es importante evaluar el desempeño de los trabajadores, de esta manera, se podrá elaborar planes de mejora continua para alcanzar una mayor productividad y rentabilidad; también nos brinda resultados para mejorar los procesos de recursos humanos, mejorar los perfiles de los puestos, detectar las necesidades de mejora, identificar al personal con potencial, enriquecer los conocimientos de la organización y tomar decisiones más acertadas en las atribuciones variables e incentivos.

De la misma manera, Salgado (2018), cuyos resultados sobre el desempeño fueron óptimos, atribuyendo a los directores y/o jefes el buen trabajo de ellos; asimismo, sugirió tomar en cuenta enfáticamente las aptitudes y capacidades individuales de sus colaboradores de esta manera incrementar su desempeño en el trabajo, conociendo sus actividades y satisfaciendo obligaciones esenciales, de esta manera, creará en el trabajador emociones y ganas de superación generando un cambio en él.

En cuanto a la caracterización de la Gestión del cambio, referente a la dimensión creación de una necesidad en común, la mayoría dijo que es regular, falta unificar criterios entre los trabajadores y hacer entender que los cambios son importantes. En cambio, en la 
dimensión crear una visión de futuro mencionaron que es muy deficiente, falta motivar a los trabajadores para enfrentarse a los cambios. Con respecto a la dimensión movilización de los implicados, la mayoría opinó que es muy deficiente, porque no existen líderes que promuevan el cambio. En la dimensión cambio de sistemas y estructuras, mayoritariamente dijo que es muy deficiente, porque no se adaptan a las innovaciones, no asumen retos. En la dimensión monitoreo de avances, la mayoría mencionó estar en desacuerdo, porque falta implementar un sistema de seguimiento, no existen evaluaciones ni cuentan con un asesor.

Según, Castillo (2016), los resultados obtenidos en su investigación son similares, puesto que la mayoría indicó que la gestión es regular, el personal busca mejorar su desempeño y asumen nuevos retos y los riesgos que se puedan presentar en el proceso de la gestión del cambio, no se encuentran capacitados para brindar un buen nivel de calidad de servicio hacia los usuarios, originando que evadan sus impuestos, arbitrios y otros, lo cual se generaría un déficit en la municipalidad.

En cambio, Pérez (2017), en los resultados de su investigación indicaron que la gestión del cambio es regular, la dirección estratégica es el procedimiento de mejora constante que permite la presentación de formas de cambio organizadas con el punto de ajustarse a las nuevas situaciones que surgen en la ejecución del sistema a pesar de mejorar la ejecución del negocio. Los elementos dinámicos se pueden agrupar en tres medidas: cultural, posicional y técnico-monetaria, y su temperamento puede ser exógeno o endógeno para la asociación. Sea como fuere, los modelos de cambio autorizados a pesar de todo necesitan incorporación, en general tendrían un alcance restringido, y no se habla de su naturaleza global.

Sin embargo, Huancaruna (2019), arguyó; es importante que una propuesta se caracterice por la participación activa entre empleados y subalternos, la libertad de expresión, el trabajo en equipo y la práctica de valores institucionales; las cuales contribuirán en elevar la cultura organizacional y promover la gestión del cambio en los trabajadores. Asimismo, se dedujo, que no tienen predisposición para asumir retos laborales; demuestran un conformismo y trabajo rutinario, por lo que hablar de cambio para algunos trabajadores les resulta difícil asumir responsabilidades que demanda nuevos aprendizajes; es decir, les resulta difícil salirse de su zona de confort. 
Los resultados de esta investigación en cuanto al desempeño laboral difieren mucho con los resultados de los antecedentes citados; puesto que los contextos y realidades son diferentes; sin embargo, fue propicio para conocer la importancia de la gestión del cambio en las organizaciones, tal como lo explica Jiménez (2012), el cambio en las estructuras institucionales, en la cultura, en la mentalidad de las personas, es una forma de interactuar tanto formal como informal, intercambiando información que repercutirá en la transformación del individuo. En cambio, para Robbins (2009), la gestión del cambio, es hacer las cosas de manera diferente en una organización. Algunas, tratan el cambio como algo accidental, este es estudiado como una actividad intencional orientada al logro de las metas.

Finalmente, todo cambio en una organización y/o compañía es necesario, puesto que trae nuevos conocimientos, saca a los trabajadores de su zona de confort enfrentándoles a nuevos retos laborales; que este, a su vez, causa malestar y desorden en un principio, pero a medida que los procesos de cambio se vayan consolidando se podrá evidenciar a través del desempeño laboral y el cumplimiento de metas. Por ello, se propone un modelo de gestión del cambio para mejorar el rendimiento laboral en las entidades municipales, partiendo desde la premisa motivacional en la que, el Estado ajuste la normativa jurídica a la realidad social, en el cual las instituciones públicas como privadas, reciban una remuneración justa y equitativa.

\section{CONCLUSIONES.}

5.1. El nivel de rendimiento laboral de los colaboradores en la Municipalidad Provincial de Rioja es regular, lo que demuestra que los trabajadores no están cumpliendo eficazmente con sus funciones.

5.2. La falta de motivación y de líderes que promuevan los cambios, impiden que los colaboradores nombrados no estén preparados para las nuevas innovaciones y la asunción de retos.

5.3. Desde la percepción de los funcionarios y colaboradores coinciden en afirmar que el desempeño laboral de los trabajadores es deficiente, es decir, la mayoría de ellos no cumple eficiente su labor, son reacios al cambio y trabajan con desgano.

5.4. No se evidencia una buena gestión del cambio, ni de líderes que lo promuevan, debido tal vez, a que los colaboradores nombrados no están preparados para las 
nuevas innovaciones y asumir retos. Asimismo, no existe un plan o programa de integración que permita capacitar a todo el personal.

5.5. Se propuso un modelo de gestión del cambio para mejorar el rendimiento laboral de los colaboradores en la Municipalidad, cuyos pilares fueron la claridad, exactitud, precisión, pertinencia y profundidad; fundamentado en lo axiológico, pedagógico, psicológico, filosófico y epistemológico.

\section{REFERENCIAS BIBLIOGRAFICAS}

Accerto. (2016). Gestión del cambio y la incertidumbre. Barcelona: Cedro.

Alles, M., (2006). Desempeño por competencias evaluación de $360^{\circ}$. Primera Edición. Buenos Aires, Argentina: Ediciones Granica S.A

Álvarez, B., Alfonso, D. \& Indacochea, B. (2018), El desempeño laboral: Un problema social de la ciencia. (artículo científico). Universidad del Pinar del Río, Cuba. Rev. Didasc@lia: Didáctica y Educación. Vol. IX, n²

Bohlander, G., \& Snell, S. (2008). Administración de recursos humanos (14 ed.). México: Thomson.

Carrasco (2016). Metodología de la investigación científica. Lima, Perú. Editorial: San Marcos E.I.R.L.

Chiavenato, I. (2011). Administración de recursos humanos (9 ed.). México: McGrawHill/Interamericana Editores.

Chiavenato, I. (2015). Comportamiento Organizacional (3 ed.). México: Mc Graw Hill.

Dessler, G. (2009). Administración de recursos humanos (11 ed.). México: Pearson Educación.

Hernández, J. A., Gallarzo, M. \& Espinoza, J. D. J. (2011). Desarrollo organizacional: un enfoque latinoamericano. México: Pearson Educación

Huancaruna, W. (2019), Modelo de gestión del cambio para mejorar la cultura organizacional en la Municipalidad provincial de Rioja, región San Martín - 2018. (Tesis doctoral). Universidad César Vallejo. Tarapoto, Perú.

Jiménez, C. (2012). Gestión del cambio organizacional. Recuperado desde: http://www.eumed.net/ce/2012/cnj.pdf

Koontz, H., Weihrich, H. y Cannice, M. (2012) Administración, una perspectiva global, Editorial Mc Graw Hill, 14va edición, México 
Lee, L. (2008). Gestión de la empresa. Recuperado desde: http://www.geocities.ws/angelcontrerasna/gestion/foro3.html

Mazabel, C. (2011). Indicadores de gestión en recursos humanos y su impacto económico en la organización "de lo cualitativo a lo cuantitativo" (1 ed.). Lima: Impresión Tarea Asociación Gráfica Educativa.

Mazariegos, R. (2016). Proyección cultural: una herramienta metodológica para la socialización de la cultura. Artes unicach 1 y 2 (10): 6-16.

Peña, E. (2018), Resistencia al cambio y trabajo en equipo. (Artículo científico). Universidad Norbert Wiener. Lima, Perú.

Pérez, L. (2017), El cambio organizacional como herramienta para coadyuvar con la implementación de la estrategia. (Artículo científico). Universidad de Holguín, La Habana, Cuba

Robbins, S. (2009). Fundamentos de la administración, conceptos esenciales y aplicaciones. (Sexta edición). México D.F.: Prentice Hall Hispanoamericana.

Robbins, S., \& Judge, T. (2009). Comportamiento Organizacional. México: Prentice Hall.

Robbins, S., y Judge, T., (2013). Comportamiento organizacional, Tercera edición, México DF, México: Pearson Educación de México.

Salgado, F. (2018), Actitud y resistencia al cambio organizacional en trabajadores mineros. (Artículo científico). Universidad de Atacama, Chile

Schermerhorn, J. (2005). Organizational Behavior. (Novena edición). New Jersey, USA: John Wiley \& Sons Inc.

Schermerhorn, J. (2005). Organizational Behavior. (Novena edición). New Jersey, USA: John Wiley \& Sons Inc.

Soriano, R. (2017), Gestión del cambio: una propuesta metodológica desde el proceso estratégico. (Revista científica). Pontifica Universidad Católica del Perú. Lima

Soto, S. R. (2001). Del origen y evolucion de la teoría de la administración: Hacia el desarrollo de nuevos modelos organizativos. Gestión y estrategias, 112 -117.

Stephen P., R., \& Timothy A., J. (2009). Comportamiento Organizacional. México: PEARSON EDUCACION

Stoner, J., Freeman, E., \& Gilbert, D. (2009). Comportamiento Organizacional (13 ed.). México: Pearson Educación. 
Toledo, M. (2018), Gestión del cambio y compromiso organizacional. (Artículo científico). Universidad Nacional, "Santiago Antúnez de Mayolo”, Huaraz, Perú.

Torres-Salazar, P. \& Melamed-Varela, E. (2016). Fundamentos teóricos de la cultura desde la óptica de las organizaciones. Desarrollo Gerencial, 8(1), 143-164. https://doi.org/10.17081/dege.8.1.1411

Wiemann, O. (2011). La comunicación en las relaciones interpersonales. . España: Editorial Aresta y UOC 